\title{
Preferences for prenatal tests for Down syndrome: an international comparison of the views of pregnant women and health professionals
}

\author{
Melissa Hill ${ }^{1}$, Jo-Ann Johnson ${ }^{2}$, Sylvie Langlois ${ }^{3}$, Hyun Lee ${ }^{3}$, Stephanie Winsor ${ }^{4}$, Brigid Dineley ${ }^{4}$, \\ Marisa Horniachek ${ }^{4}$, Faustina Lalatta ${ }^{5}$, Luisa Ronzoni ${ }^{5}$, Angela N Barrett ${ }^{6}$, Henna V Advani ${ }^{6}$, \\ Mahesh Choolani $^{6}$, Ron Rabinowitz ${ }^{7}$, Eva Pajkrt ${ }^{8}$, Rachèl V van Schendel ${ }^{9}$, Lidewij Henneman ${ }^{9}$, \\ Wieke Rommers ${ }^{10}$, Caterina M Bilardo ${ }^{10}$, Paula Rendeiro ${ }^{11}$, Maria João Ribeiro ${ }^{12}$, José Rocha ${ }^{11,12}$, \\ Ida Charlotte Bay Lund ${ }^{13}$, Olav B Petersen ${ }^{14}$, Naja Becher ${ }^{14}$, Ida Vogel ${ }^{14}$, Vigdis Stefánsdottir ${ }^{15}$, \\ Sigrun Ingvarsdottir ${ }^{16,17}$, Helga Gottfredsdottir ${ }^{16,17}$, Stephen Morris ${ }^{18}$ and Lyn S Chitty ${ }^{1}$
}

Non-invasive prenatal testing is increasingly available worldwide and stakeholder viewpoints are essential to guide implementation. Here we compare the preferences of women and health professionals from nine different countries towards attributes of non-invasive and invasive prenatal tests for Down syndrome. A discrete choice experiment was used to obtain participants' stated preference for prenatal tests that varied according to four attributes: accuracy, time of test, risk of miscarriage, and type of information. Pregnant women and health professionals were recruited from Canada, Denmark, Iceland, Israel, Italy, the Netherlands, Portugal, Singapore, and the United Kingdom. A total of 2666 women's and 1245 health professionals' questionnaires were included in the analysis. Differences in preferences were seen between women and health professionals within and between countries. Overall, women placed greater emphasis on test safety and comprehensive information than health professionals, who emphasised accuracy and early testing. Differences between women's and health professionals' preferences are marked between countries. Varied approaches to implementation and service delivery are therefore needed and individual countries should develop guidelines appropriate for their own social and screening contexts.

European Journal of Human Genetics (2016) 24, 968-975; doi:10.1038/ejhg.2015.249; published online 18 November 2015

\section{INTRODUCTION}

Many countries have established prenatal screening programmes for Down syndrome (DS), where an initial screening test is followed by the offer of an invasive diagnostic test for women with a 'high risk' result to allow definitive diagnosis. Non-invasive prenatal testing (NIPT), which analyses cell-free DNA in maternal plasma, is rapidly transforming prenatal testing for DS worldwide. ${ }^{1}$ NIPT can be used from 10 weeks in pregnancy to screen for DS with high sensitivity (99.3\%) and specificity (99.8\%) and can detect other common chromosomal aneuploidies (Trisomy 18, Trisomy 13, and Monosomy $\mathrm{X}){ }^{2,3}$ NIPT allows screening for these conditions with much greater specificity than traditional DS screening (DSS) ${ }^{4}$ and thereby significantly reduces the need for invasive testing (chorionic villus sampling or amniocentesis) with the associated small miscarriage risk. ${ }^{5}$
Following the first evidence in 2008 that NIPT for DS was feasible, ${ }^{6,7}$ this test has moved swiftly into clinical practice with testing available in the private sector since 2011. Several USA and Hong Kong/China based companies now offer NIPT and have made their commercial tests widely available. ${ }^{8}$ Uptake of NIPT, although frequently high, does vary between and within countries and a better understanding of what drives these differences is crucial to inform and optimise the prenatal testing services that are offered to women. Stakeholder viewpoints are essential to guide widespread implementation, especially in countries where NIPT will become part of a public healthcare programme. Here we have used a discrete choice experiment (DCE) to determine the relative importance placed on specific attributes of prenatal tests by pregnant women and health professionals (HPs) from different countries. By asking participants to

${ }^{1}$ Genetics and Genomic Medicine, UCL Institute of Child Health and Great Ormond Street Hospital for Children NHS Foundation Trust, London, UK; ${ }^{2}$ Department of Obstetrics \& Gynecology, University of Calgary, Calgary, Alberta, Canada; ${ }^{3}$ Department of Medical Genetics, University of British Columbia, Vancouver, British Columbia, Canada; ${ }^{4}$ Department of Obstetrics and Gynecology, McMaster University, Hamilton, Ontario, Canada; ${ }^{5}$ Clinical Genetics Unit, Fondazione IRCCS Cà Granda Ospedale Maggiore Policlinico, Milano, Italy; ${ }^{6}$ Department of Obstetrics and Gynaecology, Yong Loo Lin School of Medicine, National University of Singapore, Singapore, Singapore; ${ }^{7}$ Department of Obstetrics and Gynecology, Shaare Zedek Medical Centre, Jerusalem, Israel; ${ }^{8}$ Fetal Medicine Unit, Department of Obstetrics and Gynaecology, Academic Medical Centre, Amsterdam, The Netherlands; ${ }^{9}$ Department of Clinical Genetics, Section of Community Genetics, EMGO Institute for Health and Care Research, VU University Medical Center, Amsterdam, The Netherlands; ${ }^{10}$ Department of Obstetrics and Gynecology, Fetal Medicine Unit, University of Groningen, University Medical Centre Groningen, Groningen, The Netherlands; ${ }^{11}$ CGC Genetics, Porto, Portugal; ${ }^{12}$ IINFACTS, CESPU, Porto, Portugal; ${ }^{13}$ Department of Clinical Genetics, Aarhus University Hospital, Aarhus, Denmark; ${ }^{14}$ Department of Obstetrics and Gynecology, Aarhus University Hospital, Aarhus, Denmark; ${ }^{15}$ Department of Genetics and Molecular Medicine, Landspitali University Hospital, Reykjavik, Iceland; ${ }^{16}$ Landspitali, National University Hospital, Reykjavik, Iceland; ${ }^{17}$ Department of Midwifery, Faculty of Nursing, University of Iceland, Reykjavik, Iceland; ${ }^{18}$ Research Department of Applied Health Research, University College London, London, UK

${ }^{*}$ Correspondence: Dr M Hill, Genetics and Genomic Medicine, UCL Institute of Child Health and Great Ormond Street Hospital for Children NHS Trust, Level 5, Barclay House, 37, Queen Square, London WC1N 3BH, UK, Tel: +44 (0) 2077626871; Fax: +44 (0) 2078138578; E-mail: melissa.hill@ucl.ac.uk

Received 27 July 2015; revised 6 September 2015; accepted 23 October 2015; published online 18 November 2015 
choose between a series of hypothetical options with varying attributes, DCEs reveal which attributes influence choice behaviour, establish an individual's willingness to trade-off one attribute against another, and provide insight into real-life decision making. ${ }^{9}$ DCEs have been used to explore preferences for screening and diagnostic tests for DS, looking at attributes such as miscarriage risk, the timing of the test, and the type of information available from test results. ${ }^{9-15}$ Our previous DCE looking at preferences for prenatal tests with reference to NIPT in the United Kingdom demonstrated that HPs placed greater emphasis on test accuracy, while women prioritised test safety. ${ }^{13}$ Here we explore women's and HPs' preferences for key attributes of the current DSS and testing pathway and compare these with attitudes towards NIPT in nine culturally diverse countries with differing healthcare systems. In addition, we examine the value placed on tests that give more comprehensive information as we move from karyotyping or microarray analysis, which detects trisomies and other pathogenic cytogenetic rearrangements, to NIPT which currently only reliably detects the major trisomies.

\section{METHODS}

Study design and analysis followed DCE guidelines. ${ }^{16,17}$ Nine countries participated: Canada, Denmark, Iceland, Israel, Italy, the Netherlands, Portugal, Singapore, and the United Kingdom (Supplementary Table S1). Local ethics approval was gained by each participating research team (Supplementary Table S2).

\section{Study sample}

A convenience sample of pregnant women attending maternity clinics or midwifery practices for routine clinical care was recruited. Women were invited to complete a hard copy or online version (Denmark only) of the questionnaire while waiting for a clinic appointment and had the option of reading a brief information sheet that described DS and tests for DSS and diagnosis.

HPs, who were likely to discuss prenatal testing options when delivering antenatal care to women, were recruited. In Denmark, Iceland, Israel, and Singapore HPs were invited by email and used an online version of the questionnaire. In all other countries potential participants were approached in person or as a group at a meeting/training session and invited to complete a hard copy of the questionnaire.

The type of hospital where participants were recruited varied between and within countries and included both large academic teaching hospitals and smaller regional hospitals (Supplementary Table S3). Only one country, Singapore, recruited private patients.

\section{Questionnaire design}

The questionnaire comprised three sections: (1) structured questions about prenatal testing; (2) DCE choice sets; and (3) demographic questions. Section (1) included a question asking participants to choose between test 1 (comparable to NIPT), test 2 (comparable to invasive testing) or no test. Test 1 was described as $99 \%$ accurate testing for DS, Edward's syndrome, or Patau's syndrome, and a blood test with no risk of miscarriage but, if positive, an invasive test, which has a $1 \%$ risk of miscarriage, would be recommended. Test 2 was described as 100\% accurate testing for DS, Edward's syndrome, or Patau's syndrome, and gives additional information about rarer conditions that may cause learning disability, developmental delay, or other health problems. It was also described as being invasive with a $1 \%$ risk of miscarriage.

DCE attributes were the same as our previous UK DCE, which were obtained following literature review and consultation with stakeholders and were shown to generate plausible results. ${ }^{13}$ The attributes cover key differences between NIPT and invasive tests; accuracy, time of test, risk of miscarriage, and type of information. The associated levels reflect current clinically feasible ranges (Figure 1a). Review of the literature shows that risk of procedure related

a

\begin{tabular}{ll}
\hline Attribute & Levels \\
\hline Accuracy & $95 \%, 99 \%, 100 \%$ \\
Time of results & $10,12,16$ \\
(gestation in weeks) & Small risk (1\%), No risk \\
Risk of miscarriage & $\begin{array}{l}\text { Simple information } \\
\text { Information about Down's syndrome (T21), Edward's syndrome (T18) and } \\
\text { Patau's syndrome (T13) only. }\end{array}$ \\
Information from test & $\begin{array}{l}\text { Comprehensive information } \\
\text { Information about Down's syndrome (T21), Edward's syndrome (T18) and } \\
\text { Patau's syndrome (T13) and information about rare conditions that may } \\
\text { cause learning disability, developmental delay or other health problems. }\end{array}$ \\
\hline
\end{tabular}

b

\begin{tabular}{l|ll}
\hline Choice 1 & Test A & Test B \\
\hline Accuracy & $95 \%$ & $100 \%$ \\
Time of results & 9 weeks & 11 weeks \\
Risk of miscarriage & Small risk $(1 \%)$ & No risk \\
Information from test & Comprehensive information & Simple information \\
\hline
\end{tabular}

Which test would you prefer (tick one box only)?

Test A $\quad$ Test B $\square \quad$ Neither $\square$

Figure 1 Design of the discrete choice experiment. (a) Attributes and levels used in the discrete choice experiment. (b) Example of a discrete choice experiment choice set. 
miscarriage is $0.5-1 \%$ for amniocentesis and chorionic villus sampling, for simplicity the value of $1 \%$ was chosen for the scenarios in the questionnaire. ${ }^{18}$

The DCE design follows the approach of Street and Burgess. ${ }^{16}$ Two attributes had three levels and two attributes had two levels. The possible combinations of attributes and levels was statistically reduced from $32\left(2^{3} \times 2^{2}\right)$ to 9 scenarios, using an orthogonal fractional main effects design. ${ }^{19} \mathrm{~A}$ shift of one level created nine additional scenarios. The two sets of scenarios were paired to form nine choice sets. An additional choice set with one clearly superior test was included as an internal consistency check. Across the choice sets, all levels of each attribute occur with equal frequency (level balance) and within each individual choice set there is no overlap in attribute levels (minimal overlap). Women were asked which test they would prefer to have and HPs were asked which test they would prefer to offer. Participants were asked to choose between test A, test B or Neither (Figure 1b).

Demographic questions for women included age, gestational age, education, religion, number of children, number of children with DS, familiarity with children with DS, and interest in prenatal testing. Demographic questions for HPs included job title, years in role, age, and gender. For non-English speaking countries, the questionnaires were translated by the local research teams.

\section{Analysis}

The DCE preference data were analysed for both women and HPs using a conditional logit regression model. ${ }^{20} \mathrm{~A}$ constant term was included in the model to reflect the 'neither' option. ${ }^{21}$ The sign $(+$ or -$)$ of the coefficients generated in the regression analysis indicates the direction of the preference for each attribute. Participants were anticipated to prefer tests with greater accuracy, information, and safety ( + coefficient) conducted early in pregnancy (- coefficient). As the different attributes do not have the same unit of change, the absolute value of the coefficients has no direct interpretation. Each attribute must be measured on a common scale to enable comparison, this can be done in a number of ways including calculating the marginal rates of substitution and predicted probability analysis. ${ }^{17}$ The marginal rates of substitution were calculated as a ratio of the coefficients of two attributes to allow direct assessment of how much of one attribute participants were willing to trade-off for more of another attribute. We also determined the predicted probability that two tests from the choice set representative of NIPT and invasive testing would be selected based on the model coefficients. ${ }^{17}$ The software package Stata 12.0 (StataCorp, College Station, TX, USA) was used to perform all analyses.

\section{RESULTS}

\section{Participants}

Overall, 2707 women and 1275 HPs were recruited from 9 countries. Questionnaires were excluded if the consistency question was not answered as expected or if the respondents did not complete the choice set (women $n=41$; HPs $n=30$ ). Consequently, a total of 2666 women's and 1245 HPs' questionnaires were included in the analysis (Supplementary Table S3). The sample consisted of a highly educated group of women with over half having degree-level education in seven countries (Supplementary Table S4). In Italy the mean age was 36.2 years compared with 27.0-31.4 years for other countries. DSS uptake/ interest varied between countries from 90.3 to $97.4 \%$ in Denmark, Iceland, Italy, and Portugal, 78.6-81.6\% in Canada, Singapore, and the United Kingdom, to $69.8 \%$ in Israel and $46.0 \%$ in the Netherlands. For HPs, the mean age ranged from 34.5 years in Portugal to 47.7 years in Iceland (Supplementary Table S5). Training background varied widely between countries as DSS counselling is delivered by different professionals. In all countries the vast majority of HPs were women.

\section{Comparison of all women and HPs}

All attributes had a significant impact on women's and HPs' decision making; positive coefficients show that participants prefer safer tests with greater accuracy and comprehensive information, while the negative coefficient indicates preference for an earlier test (Table 1). 
These results are consistent with a priori expectations, supporting the theoretical validity of the models. Comparison of women and HPs demonstrate that women assign a relatively higher value to test safety and having comprehensive information, while HPs place more emphasis on accuracy and early testing than women do (Table 1). Probability analysis suggests women and HPs were equally likely to choose NIPT, but HPs would be more likely to choose invasive testing than women (Table 1). The marginal rates of substitution confirmed women's strong preference for a test with no risk of miscarriage as they were prepared to wait more than twice as long and accept 6 percentage points lower accuracy compared with HPs for a test that had no miscarriage risk (Table 2). Women were prepared to wait more than twice as long and accept a 2 percentage point decrease in accuracy compared with HPs for a test that gave comprehensive information.

Comparison of women and HPs within countries

Comparison of women and HPs identified differences in preferences within each of the countries (Tables 3 and 4). In the majority of countries women valued safety more than HPs, whereas HPs placed more emphasis on accuracy and early testing than women. In three countries, Canada, the Netherlands and Portugal, women placed more emphasis on having comprehensive information than HPs (Tables 3 and 4).

\section{Comparison of women between countries}

This comparison identified differences in preferences for each test attribute (Table 4). Notably, women from Iceland, the Netherlands, and UK placed greater emphasis on test safety than women from other countries, whereas Italian women placed much lower emphasis on safety than all other countries. The mean probability of choosing a test with the attributes similar to either NIPT or invasive testing suggest that women from Italy and Portugal and, to a lesser extent, Israel, and Singapore, were more prepared to accept tests with a miscarriage risk to gain more comprehensive information than women from other countries (Figure 2).

\section{Comparison of HPs between countries}

The preferences of HPs also differed between countries for each attribute (Table 4). For example HPs in the Netherlands placed the greatest value on test accuracy and HPs in the Netherlands, Canada, and the United Kingdom placed greater value on test safety than HPs from other countries. The mean probability of choosing a test with the attributes similar to either NIPT or invasive testing suggest that HPs from Israel, Italy, Portugal, and Singapore are more likely to prefer to offer tests with a miscarriage risk to gain more comprehensive information (Figure 2).

\section{Comparison of all women by age}

Comparison of all women 35 and older with those under 35 identified differences in preferences, with older women placing greater value on having comprehensive information and less value on safety than younger women (Supplementary Table S6).

\section{Direct choice between NIPT, invasive testing or no test}

When asked to choose directly between having NIPT, invasive testing or no test, the majority of women (79.7-56.0\%) in Denmark $(79.7 \%)$, Canada (56.0\%), Iceland (62.8\%), Israel (37.7\%), the Netherlands (47.7\%), Singapore (46.32\%), and UK (60.3\%) chose NIPT over invasive testing (Figure 3a). In Italy and Portugal more women (51.7 and $59.1 \%$ respectively) chose invasive testing rather than NIPT. Most countries had a sizeable proportion of women who chose not to have testing, including more than one third of women in the Netherlands and Israel. With the exception of Portugal, the majority of HPs in each country chose NIPT as their preferred test (Figure 3b). In Israel, Italy, Portugal, Singapore, and UK a sizeable proportion (30\% or more) chose invasive testing. The proportion of HPs choosing no test was very small $(0-11.5 \%)$.

Table 2 Marginal rates of substitution comparing all women and health professionals

\begin{tabular}{|c|c|c|c|c|}
\hline & \multicolumn{2}{|c|}{ Number of weeks respondents are prepared to wait } & \multicolumn{2}{|c|}{ Reduction in accuracy (\%) respondents are prepared to accept } \\
\hline & Women & Health professionals & Women & Health professionals \\
\hline Test with no risk of miscarriage & $13.56(1.465 /-0.108)$ & $5.57(0.986 /-0.177)$ & $9.21(1.465 / 0.159)$ & $3.13(0.986 / 0.315)$ \\
\hline Test with comprehensive information & $5.09(0.550 /-0.108)$ & $2.36(0.418 /-0.177)$ & $3.46(0.550 / 0.159)$ & $1.33(0.418 / 0.315)$ \\
\hline Test with $5 \%$ greater accuracy & $7.36(0.159 /-0.108 \times 5)$ & $8.90(0.315 /-0.177 \times 5)$ & - & - \\
\hline Earlier test & - & - & $0.68(-0.108 / 0.159)$ & $0.56(-0.177 / 0.315)$ \\
\hline
\end{tabular}

Table 3 Summary results for the conditional logit regression analysis comparing women and health professionals between countries

\begin{tabular}{|c|c|c|c|c|}
\hline & Accuracy & Time of results & No risk of miscarriage & Comprehensive information \\
\hline Canada & $\mathrm{HP}$ & $\mathrm{HP}$ & Women & Women \\
\hline Denmark & HP & No difference & Women & No difference \\
\hline Iceland & No difference & HP & Women & No difference \\
\hline Israel & HP & HP & Women & No difference \\
\hline Italy & HP & No difference & No difference & No difference \\
\hline Netherlands & HP & $\mathrm{HP}$ & Women & Women \\
\hline Portugal & HP & No difference & Women & Women \\
\hline Singapore & HP & $\mathrm{HP}$ & Women & No difference \\
\hline UK & HP & $\mathrm{HP}$ & Women & No difference \\
\hline
\end{tabular}

The table shows where there was a significant difference between women and HPs in the emphasis placed on the attribute during decision making and whether it was women or HPs who had the strongest preference for the attribute. 
Table 4 Conditional logit regression and probability analysis comparing women and health professionals within and between countries

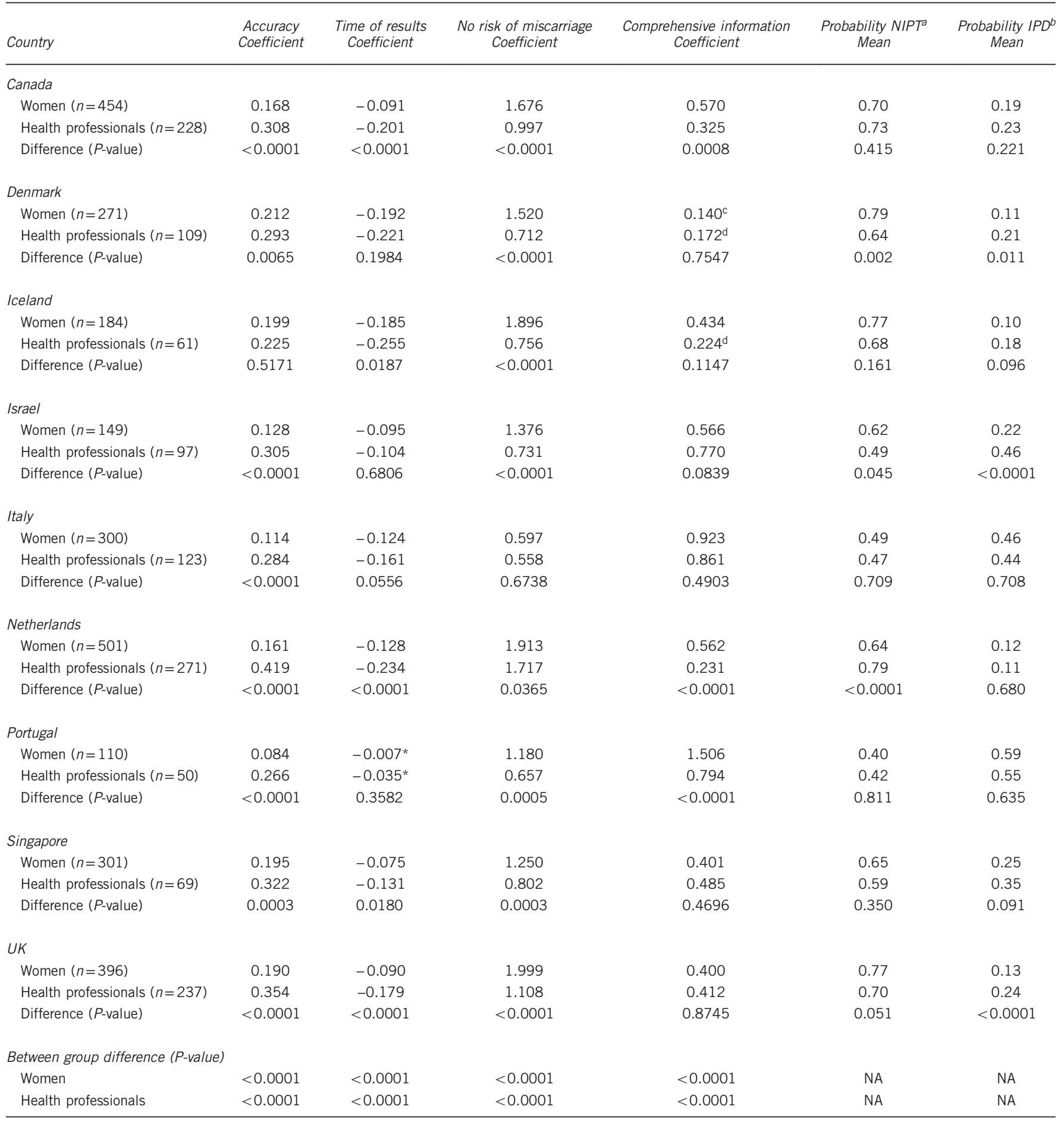

Abbreviations: IPD, invasive prenatal diagnosis; NA, not applicable; NIPT, non-invasive prenatal test.

aDefined as having the following attributes: accuracy $=99 \%$; time of results (gestation in weeks) $=12$; risk of miscarriage =none; information gained from the test $=$ simple information.

${ }^{b}$ Defined as having the following attributes: accuracy $=100 \%$; time of results (gestation in weeks) $=16$; risk of miscarriage $=$ small risk $(1 \%)$; information gained from the test $=$ comprehensive information

${ }^{c}$ Coefficient significant $P<0.05$. All other coefficients significant $P<0.0001$.

${ }^{d}$ Coefficient not significant.

\section{DISCUSSION}

Much has been published on NIPT, with many professional bodies advocating implementation for high-risk women, and others proposing its use as a primary screen, replacing traditional DSS. ${ }^{22}$ With NIPT now available through commercial companies in more than 60 countries $^{8}$ and evaluation of implementation in the public sector underway, ${ }^{23-25}$ this exploration of cross-cultural variation in women's and HPs' preferences for prenatal tests is timely. Overall, women 


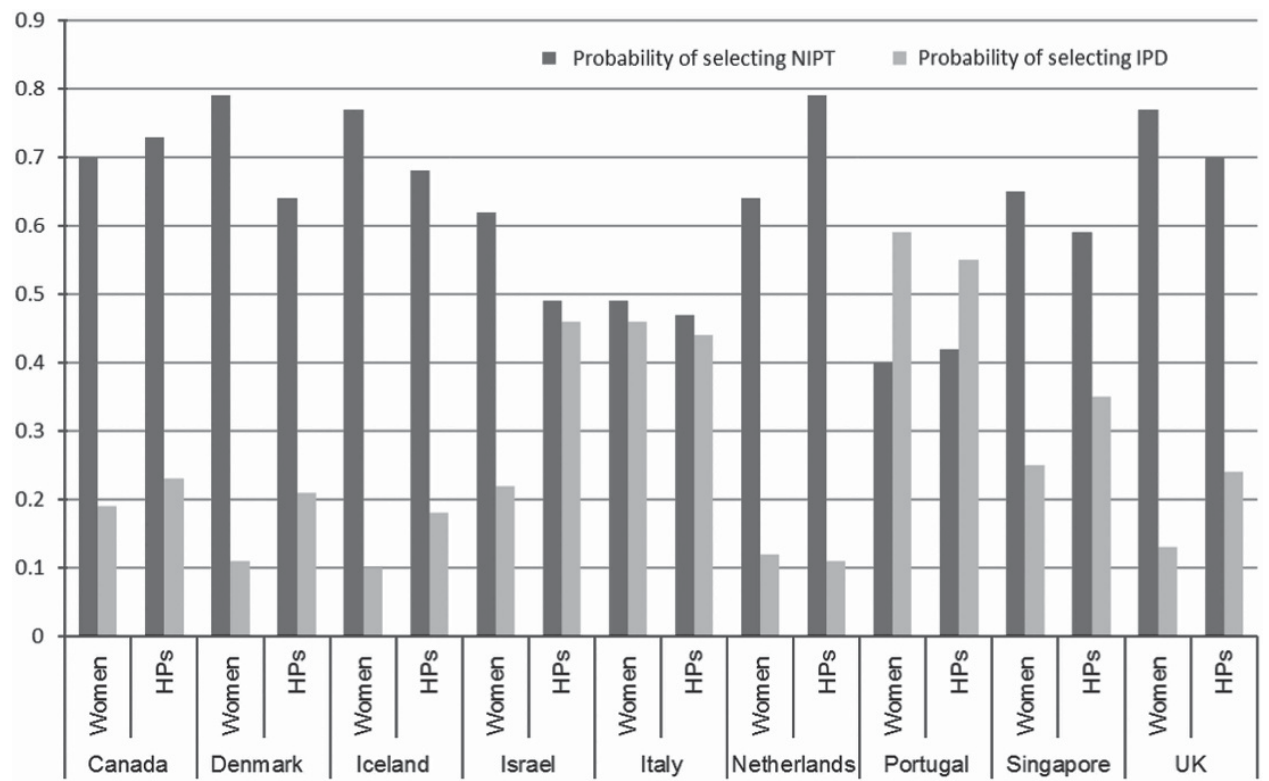

NIPT (non-invasive prenatal testing): Defined as having the following attributes: accuracy $=99 \%$; time of results (gestation in weeks) $=12$; risk of miscarriage (

IPD (invasive prenatal diagnosis): Defined as having the following attributes: accuracy $=100 \%$; time of results (gestation in weeks) $=16$; risk of miscarriage $=$ small risk (1\%); information gained from the test = comprehensive information

Figure 2 Probability that women and health professionals from each country would choose the tests from the choice sets that most closely resemble NIPT or IPD.
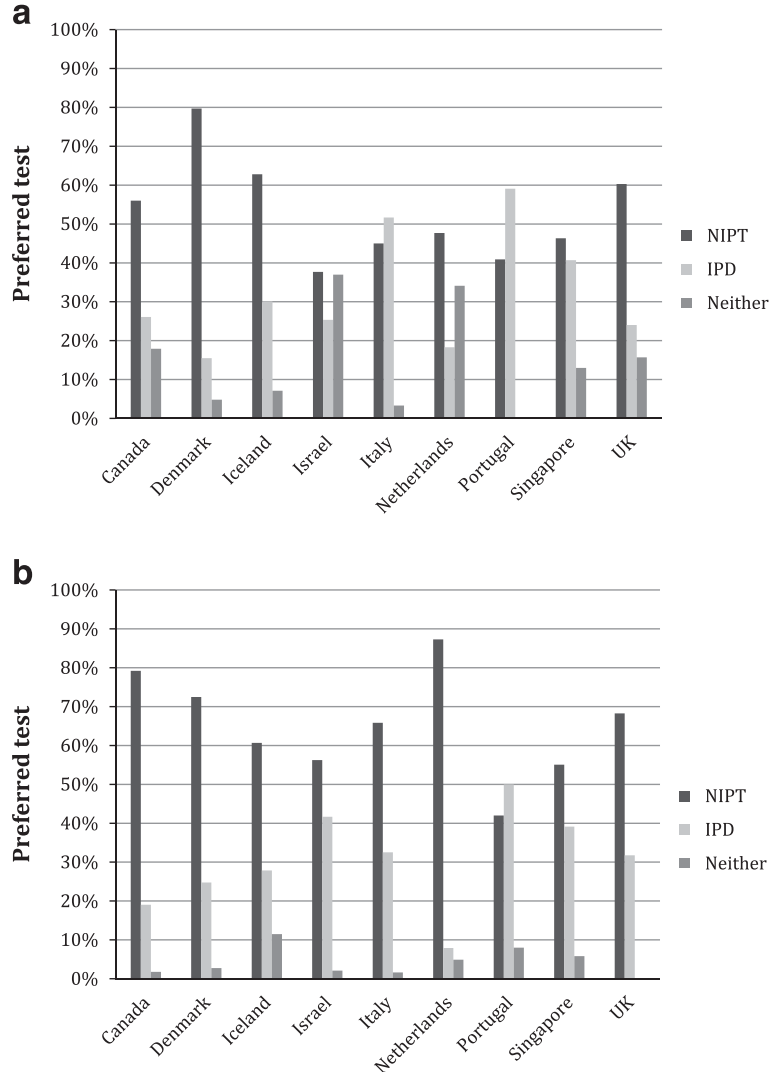

NIPT: Non-invasive prenatal test; IPD: Invasive prenatal diagnosis

Figure 3 Direct choice between test 1 (NIPT), test 2 (IPD) and no test. (a). Women's preferred test for Down syndrome. (b). Health professional's preferred test for Down syndrome. placed greater emphasis on test safety and having comprehensive information than HPs, who placed more emphasis on accuracy and early testing than women. Differences were seen between women and HPs within an individual country and there were clear differences between women's and HPs' preferences between countries. These findings highlight the need for implementation strategies of NIPT to be country specific. For example, women from Italy and Portugal placed stronger emphasis on having comprehensive information on all chromosomal rearrangements than women from other countries, implying greater demand for the provision of invasive testing in these countries. Ideally, further targeted research into stakeholder views should be used to inform individual approaches to service delivery.

Previous DCE studies looking at prenatal testing for DS in the United Kingdom, ${ }^{9,10,15}$ Australia, ${ }^{11}$ and the Netherlands ${ }^{14}$ have shown similar results, whereby HPs valued accuracy and earlier timing of tests while women emphasised test safety and information. This difference is important, and HPs need to be aware that their views may differ from the women they are counselling. In all countries, pregnant women were willing to accept a less accurate prenatal test to access a test without miscarriage risk. To promote informed decision making, HPs should not focus solely on the benefit of test safety when discussing NIPT but should also carefully address the limitations of current NIPT technology. Informed decision making is an active process that in this setting should include accurate and relevant information about the scope and limitations of the tests, appropriate and up-to-date information about the conditions that are being tested for, and an opportunity to deliberate on all information in light of individual values and beliefs. ${ }^{26}$ How HP's promote informed decision making is particularly pertinent as HPs determine how tests are presented and influence test uptake, ${ }^{27}$ and women reportedly follow the advice of trusted HPs. ${ }^{28}$ The option of not having testing also needs to be highlighted to women. When asked directly to choose between NIPT, invasive testing and no test, a sizeable proportion of 
women, particularly in Israel and the Netherlands, chose no test. In Israel cultural factors may have influenced this outcome as participants included a large proportion of Orthodox Jewish women and in the Netherlands attitudes to testing may influence DSS uptake. Our findings mirror results of questionnaire studies conducted in the Netherlands ${ }^{29}$ and USA, ${ }^{30}$ which found that even with NIPT as an option, up to one third of participants would decline DSS.

Our results suggest that values attached to key attributes of prenatal tests differ across countries for both women and HPs. Previous research has shown variation in women's views on prenatal testing between countries with respondents from Northern European countries more likely to value parental choice in prenatal testing than their counterparts in Southern Europe and Asia. ${ }^{31}$ Variation in women's preferences between countries raises the question of whether differences exist between cultural groups within countries. There is some evidence suggesting ethnic minority groups in Western countries differ in how they view and use prenatal screening and testing. ${ }^{32-34}$ Further research is needed to determine whether these differences include attitudes to NIPT and, if so, how these could be addressed to improve service delivery in increasingly multicultural societies.

Differences between countries are likely to reflect personal decisions influenced by attitudes to prenatal testing, disability, and termination and wider influences such as religion, social, and cultural contexts and healthcare policies such as the requirement for part or full payment for prenatal testing and access to termination of pregnancy. More nuanced differences in DSS programmes and policies may also impact on preferences. Crombag et al. ${ }^{35}$ compared DSS programmes in Denmark, the Netherlands, and UK and speculated that variation in DSS uptake rates between these countries are influenced by how the offer of DSS is framed and viewed. For example, in Denmark (highest uptake) DSS is free and is offered in a social context where screening is viewed by many women as a routine part of prenatal care. In the Netherlands (lowest uptake) testing must be paid for and women are first explicitly asked if they want information on DSS before the information is provided, which places clear emphasis on the right not to know. Age differences may impact on the differences observed, with older women placing more emphasis on having comprehensive information and less emphasis on safety than younger women. This may in part explain the differences seen between Italian women and those from other countries as the mean age of the women recruited from Italy was greater than those from other countries. Other factors that may have a part in the differences seen between countries could include the type of hospital where recruitment took place or whether private or public patients were recruited.

\section{Study limitations}

A number of issues may limit the general applicability of our findings. This was a convenience sample and participants were self-selected, which may result in a bias towards those interested in prenatal testing taking part in the study. In addition, the majority (55.3\%) of women who took part were highly educated and held a degree qualification or equivalent. Recruitment at individual centres may not reflect preferences across the whole country. For example, DSS uptake has been shown to vary considerably between different UK regions ${ }^{27}$ and in the Netherlands. ${ }^{36}$ In addition, the study was conducted in high-income countries. Low-income countries will face additional challenges and focused research is needed. ${ }^{8}$ Another limitation is that partner preferences were not included in the study. Their views are clearly important and may indeed differ from those of women. The DCE design only considered four attributes of prenatal tests when real-life choices would also involve other factors such as false positives, access to tests and costs, and does not explore reasoning behind the choices made or give insight into how tests were perceived. Moreover, as in any stated-preference study, participant choices do not necessarily reflect choices that would be made if participants were faced with a real-life decision about testing. Another potential limitation is that the questionnaire was developed in English with translation undertaken by the local research team. The translation process may have resulted in variations in meaning and interpretation of the questionnaire and there may also be a loss of culturally specific meanings. ${ }^{37}$

\section{CONCLUSION}

Differences between women's and HPs' preferences were marked between countries, making it clear that approaches to NIPT implementation and service delivery should be country specific. Accordingly, it is important that individual countries take time to research implementation strategies, gather stakeholder views, and develop guidelines appropriate for their own social and screening contexts. Within each country women and HPs differed in the value they placed on test attributes. Implementation policies need to consider these differences to ensure needs of all stakeholders are met. As all women place great emphasis on test safety, it is important that pre-test counselling for NIPT thoroughly explores other attributes of NIPT, considers alternative options, and reflects on the possible implications of testing so that the focus of discussion is not just test safety.

\section{CONFLICT OF INTEREST}

The authors declare no conflict of interest.

\section{ACKNOWLEDGEMENTS}

We are grateful to the women and HPs who participated in the study. We also thank the local principal investigators and researchers at each hospital for their help with recruitment. This manuscript presents independent research funded by the National Institute for Health Research (NIHR) under the Programme Grants for Applied Research programme (RP-PG-0707-10107) (the "RAPID" project), the NIHR Comprehensive Research Network and the NIHR Biomedical Research Centre at Great Ormond Street Hospital for Children. The views expressed are those of the authors and not necessarily those of the NHS, the NIHR or the Department of Health.

1 Minear MA, Lewis C, Pradhan S, Chandrasekharan S: Global perspectives on clinical adoption of NIPT. Prenat Diagn 2015; 35: 959-967.

2 Gil MM, Quezada MS, Revello R, Akolekar R, Nicolaides KH: Analysis of cell-free DNA in maternal blood in screening for fetal aneuploidies: updated meta-analysis. Ultrasound Obstet Gynecol 2015; 45: 249-266.

3 American College of Obstetricians and Gynecologists: Committee opinion no. 640: cellfree dna screening for fetal aneuploidy. Obstet Gynecol 2015; 126: e31-e37.

4 Norton ME, Jacobsson B, Swamy GK et al: Cell-free DNA analysis for noninvasive examination of trisomy. N Engl J Med 2015; 372: 1589-1597.

5 Warsof SL, Larion S, Abuhamad AZ: Overview of the impact of noninvasive prenatal testing on diagnostic procedures. Prenat Diagn 2015; 35: 972-979.

6 Chiu RW, Chan KC, Gao Y et al: Noninvasive prenatal diagnosis of fetal chromosomal aneuploidy by massively parallel genomic sequencing of DNA in maternal plasma. Proc Natl Acad Sci USA 2008; 105: 20458-20463.

7 Fan HC, Blumenfeld YJ, Chitkara U, Hudgins L, Quake SR: Noninvasive diagnosis of fetal aneuploidy by shotgun sequencing DNA from maternal blood. Proc Natl Acad Sci USA 2008; 105: 16266-16271.

8 Allyse M, Minear MA, Berson E et al: Non-invasive prenatal testing: a review of international implementation and challenges. Int J Womens Health 2015; 7: 113-126.

9 Ryan M, Diack J, Watson V, Smith N: Rapid prenatal diagnostic testing for Down syndrome only or longer wait for full karyotype: the views of pregnant women. Prenat Diagn 2005; 25: 1206-1211.

10 Bishop A, Marteau T, Armstrong D et al: Women and health professional's preferences for Down's Syndrome screening tests: a conjoint analysis study. BJOG 2004; 111: 775-779.

11 Lewis SM, Cullinane FM, Carlin JB, Halliday JL: Women's and health professionals' preferences for prenatal testing for Down syndrome in Australia. Aust N Z J Obstet Gynaecol 2006; 46: 205-211. 
12 Lewis SM, Cullinane FN, Bishop AJ et al: A comparison of Australian and UK obstetricians' and midwives' preferences for screening tests for Down syndrome. Prenat Diagn 2006; 26: 60-66.

13 Hill M, Fisher J, Chitty LS, Morris S: Women's and health professionals' preferences for prenatal tests for Down syndrome: a discrete choice experiment to contrast noninvasive prenatal diagnosis with current invasive tests. Genet Med 2012; 14: 905-913.

14 Beulen L, Grutters JP, Faas BH et al: Women's and healthcare professionals' preferences for prenatal testing: a discrete choice experiment. Prenat Diagn 2015; 35: 549-557.

15 Carroll FE, Al-Janabi H, Flynn T, Montgomery AA: Women and their partners preferences for Down's syndrome screening tests: a discrete choice experiment. Prenat Diagn 2013; 33: 449-456.

16 Ryan M, Gerard K, Amaya-Amaya M: Using Discrete Choice Experiments to Value Health and Health Care. Dordrecht, Netherlands: Springer, 2008.

17 Lancsar E, Louviere J: Conducting discrete choice experiments to inform healthcare decision making: a user's guide. Pharmacoeconomics 2008; 26: 661-677.

18 Tabor A, Alfirevic Z: Update on procedure-related risks for prenatal diagnosis techniques. Fetal Diagn Ther 2010; 27: 1-7.

19 Hahn G, Shapiro S: A Catalogue and Computer Program for the Design and Analysis of Orthoganol Symmetric and Asymmetric Fractional Factorial Experiments. Schenectady, NY, USA: General Electric Research and Development Centre, 1966.

20 McFadden D, Conditional logit analysis of qualitative choice behavior, in Zarembka P (ed): Frontiers in Econometrics. New York, USA: Academic Press, 1974, pp 105-142.

21 Haaijer R, Kamakura W, Wedel M: The "no-choice" alternative in conjoint choice experiments. Int J Market Res 2001; 43: 93-106.

22 Dondorp W, de Wert G, Bombard Y et al: Non-invasive prenatal testing for aneuploidy and beyond: challenges of responsible innovation in prenatal screening. Eur J Hum Genet 2015; 23: 1592

23 Hill M, Wright D, Daley R et al: Evaluation of non-invasive prenatal testing (NIPT) for aneuploidy in an NHS setting: a reliable accurate prenatal non-invasive diagnosis (RAPID) protocol. BMC Pregnancy Childbirth 2014; 14: 229.

24 TRIDENT Study. Available at:. http://www.emgo.nl/research/quality-of-care/researchprojects/1451/trident-study-trial-by-dutch-laboratories-for-evaluation-of-non-invasiveprenatal-testing-nipt/background/ (accessed on April 2015).

25 PEGASUS Study. Available at:. http://pegasus-pegase.ca/pegasus/ (accessed on April 2015).
26 Hodgson J, Spriggs M: A practical account of autonomy: why genetic counseling is especially well suited to the facilitation of informed autonomous decision making. J Genet Couns 2005; 14: 89-97.

27 Dormandy E, Marteau TM: Uptake of a prenatal screening test: the role of healthcare professionals' attitudes towards the test. Prenat Diagn 2004; 24: 864-868.

28 Lewis C, Silcock C, Chitty LS: Non-invasive prenatal testing for Down's syndrome: pregnant women's views and likely uptake. Public Health Genomics 2013; 16 223-232.

29 van Schendel RV, Dondorp WJ, Timmermans DR et al: NIPT-based screening for Down syndrome and beyond: what do pregnant women think? Prenat Diagn 2015; 35 598-604.

30 Allyse M, Sayres LC, Goodspeed TA, Cho MK: Attitudes towards non-invasive prenata testing for aneuploidy among US adults of reproductive age. J Perinatol 2014; 34 $429-434$.

31 van den Heuvel A, Chitty L, Dormandy E et al: Is informed choice in prenatal testing universally valued? A population-based survey in Europe and Asia. BJOG 2009; 116 880-885

$32 \mathrm{Yu}$ J: A systematic review of issues around antenatal screening and prenatal diagnostic testing for genetic disorders: women of Asian origin in western countries. Health Soc Care Community 2012; 20: 329-346.

33 Fransen MP, Essink-Bot ML, Vogel I et al: Ethnic differences in informed decisionmaking about prenatal screening for Down's syndrome. J Epidemiol Community Health 2010; 64: 262-268.

34 Gitsels-van der Wal JT, Verhoeven PS, Mannien J et al: Factors affecting the uptake of prenatal screening tests for congenital anomalies; a multicentre prospective cohort study. BMC Pregnancy Childbirth 2014; 14: 264.

35 Crombag NM, Vellinga YE, Kluijfhout SA et al: Explaining variation in Down's syndrome screening uptake: comparing the Netherlands with England and Denmark using documentary analysis and expert stakeholder interviews. BMC. Health Serv Res 2014: 14: 437.

36 Bakker M, Birnie E, Pajkrt E, Bilardo CM, Snijders RJ: Low uptake of the combined test in The Netherlands-which factors contribute? Prenat Diagn 2012; 32 1305-1312.

37 Flaherty JA, Gaviria FM, Pathak D et al: Developing instruments for cross-cultural psychiatric research. J Nerv Ment Dis 1988; 176: 257-263.

Supplementary Information accompanies this paper on European Journal of Human Genetics website (http://www.nature.com/ejhg) 